\title{
Secagem da madeira de Hovenia dulcis e Eucalyptus tereticornis em forno micro-ondas com diferentes potências de temperatura
}

\author{
Maiara Talgatti ${ }^{1}$ Talita Baldin $^{2 *}$ Elio José Santini $^{1}$ Silviana Rosso ${ }^{3}$ Laura Hoffmann Oliveira ${ }^{1}$ \\ Guilherme Valcorte ${ }^{1}$
}

\begin{abstract}
${ }^{1}$ Universidade Federal de Santa Maria, Av. Roraima, 1000, Camobi CEP: 97105900, Santa Maria, RS, Brasil
${ }^{2}$ Universidade Federal de Minas Gerais, Av. Universitária, 1.000 - Bairro Universitário, CEP: 39.404-547, Montes Claros, MG, Brasil ${ }^{3}$ Universidade Federal do Pampa, Av. Maria Anunciação Gomes Godoy, 1650, CEP 96460-000, Bagé, RS, Brasil
\end{abstract}

\section{Original Article \\ *Corresponding author: talitabaldin@ica.ufmg.br \\ Keywords: \\ Microwave drying \\ Moisture contente \\ Drying rate \\ Moisture gradient \\ Palavras-chave: \\ Secagem em micro-ondas \\ Teor de umidade \\ Taxa de secagem \\ Gradiente de Umidade}

Received in

2019/04/13

\section{Accepted on}

2020/07/30

Published in

2020/11/02

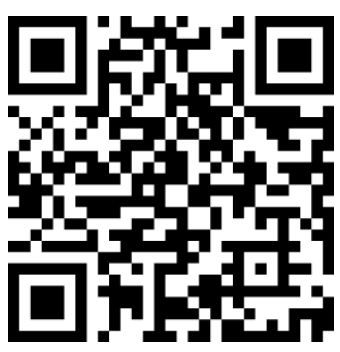

DOI: http://dx.doi.org/ 10.34062/afs.v7i3.10153

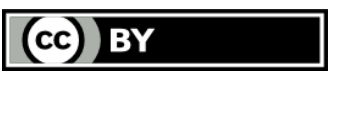

RESUMO: A secagem da madeira utilizando micro-ondas é recente no Brasil carecendo de mais pesquisas para melhorar o conhecimento e divulgação desta tecnologia. O objetivo deste estudo é avaliar a secagem da madeira em forno microondas com diferentes potências de temperatura e comparar seu uso no processo de remoção de água em Hovenia dulcis e Eucalyptus tereticornis. Amostras foram submetidas a secagem em micro-ondas com 50\% e 100\% da potência de temperatura. Avaliou-se o teor de umidade inicial, a taxa de secagem e o gradiente de umidade. Hovenia dulcis, mesmo apresentando maior teor de umidade inicial atingiu o teor de umidade final em menor tempo. A taxa de secagem para esta espécie também foi superior $(0,89$ na potência de $100 \%$, contrapondo 0,22 para $E$. tereticornis) indicando que a água flui rapidamente, mesmo com a madeira apresentando mais que o dobro de umidade em relação à E. tereticornis. Os gradientes de umidades para Eucalyptus tereticornis apresentaram valores negativos, indicando que a parte interna das peças $(-1,36 \mathrm{em} 100 \% \mathrm{e}-1,63 \mathrm{em} \mathrm{50 \% )}$ estavam com menor teor de umidade que a parte externa (-1,82 em $100 \%$ e - $-1,88 \mathrm{em}$ $50 \%$ ) refletindo uma das principais características da secagem por micro-ondas, que é o aquecimento de dentro para fora, gerando calor diretamente no centro da madeira. Em ambas as potências de temperaturas observou-se que a madeira de Eucalyptus tereticornis apresentou maior dificuldade de secagem em comparação a Hovenia dulcis.

\section{Hovenia dulcis and Eucalyptus tereticornis wood drying in microwave oven with different temperature powers}

\begin{abstract}
Microwave drying of wood is recent in Brazil, requiring more research to improve knowledge and dissemination of this technology. The objective of this study is to evaluate the drying of the wood in a microwave oven with different temperature powers and to compare its use in the water removal process in Hovenia dulcis and Eucalyptus tereticornis. Samples were subjected to microwave drying with $50 \%$ or $100 \%$ power level. Moisture content, drying rate and moisture gradient were evaluated. Hovenia dulcis, even with a higher initial moisture content, reached the final moisture content in less time. The drying rate for this species was also higher ( 0.89 at $100 \%$ power level, compared to 0.22 for E. tereticornis) indicating that water flows quickly, even with the wood presenting more than twice the humidity in relation to the E. tereticornis. Moisture gradients for Eucalyptus tereticornis showed negative values, indicating that the inside of the analyzed wood pieces (-1,36 in 100\% and $-1,63$ in 50\% power level) were drier than the outside $(-1,82$ in $100 \%$ and $-1,88$ in $50 \%$ power level), reflecting one of the main characteristics of microwave drying, which is heating from the inside out, generating heat directly in the center of the wood. Regardless of the power level used, Eucalyptus tereticornis wood presented greater difficulty in drying compared to Hovenia dulcis.
\end{abstract}




\section{Introdução}

Uma das operações mais importantes na indústria de transformação da madeira é a secagem. O conhecimento sobre técnicas que possibilitem diminuir o tempo de secagem e simultaneamente preservar as características da madeira são as chaves para reduzir o consumo de energia no processo, acarretando a redução dos custos e alavancando os lucros. Além de que, a remoção da umidade até um teor adequado, irá minimizar os efeitos da movimentação dimensional, resultando em maior qualidade do material (Santini et al. 2016).

A secagem da madeira agrega valor ao produto pois garante ao mercado consumidor um material livre de defeitos, com certo grau de resistência biológica, elevada resistência mecânica e melhor estabilidade dimensional. A escolha de um determinado método de secagem influencia no tempo, na qualidade do material seco e na obtenção do teor de umidade desejado (Susin et al. 2014).

Estudos relacionados com a secagem da madeira são indispensáveis, pois muitas das suas propriedades são sensíveis às variações de umidade abaixo do PSF, em torno de $28 \%$. Conhecer o comportamento da madeira durante a redução da umidade possibilita melhorar seu aproveitamento e sua utilização. Nesse sentido, a rapidez com que as tecnologias empregadas na secagem evoluem estão relacionadas à demanda de incentivos científicos e econômicos, além de atender às necessidades humanas, sejam estas imediatas ou futuras (Guella 2016).

As tecnologias de micro-ondas vêm sendo utilizada em nível científico e em processos industriais com o objetivo de tornar possível a rápida secagem da madeira e com elevada qualidade, reduzindo os defeitos decorrentes das tensões de crescimento, proporcionando o aumento da permeabilidade em toras e na madeira serrada, além da promoção facilitada de tratamentos preservativos de espécies refratárias ou de difícil impregnação (Torgovnikov e Vinden 2010).

Pesquisas sobre a aplicação de micro-ondas como pré-tratamento em madeiras de difícil secagem descobriram que internamente a irradiação de microondas atua estourando os vasos obstruídos por tiloses, aumentando a permeabilidade longitudinal. Além de criar microfissuras nas paredes celulares que conectam as fibras e os raios, melhorando o translato radial da água. Os resultados de tais modificações anatômicas na madeira facilitam a aceleração da taxa de secagem (Harris et al. 2008).

A metodologia de secagem com micro-ondas, além de possibilitar a remoção da água de impregnação, pode ser indicada como uma présecagem da madeira, reduzindo o tempo de permanência em estufa e permitindo maior uniformidade da umidade (Dening et al., 2000). Por outro viés, o micro-ondas também pode ser combinado com a secagem ao ar livre, acarretando maiores ganhos em relação a qualidade desse material.

Outra característica da secagem por microondas é o aquecimento de dentro para fora, gerando calor diretamente no centro da madeira (Cavalcante et al. 2016). Teoricamente, comentam os autores, o feixe de energia eletromagnética atua no núcleo do corpo a ser aquecido, apresentando um efeito que causa a pressão osmótica, ou seja, uma pressão interna como se fosse um êmbolo empurrando a água do centro para a periferia, uma vez que a vibração das moléculas é maior no interior da madeira.

Já é bem sabido que a secagem de espécies do gênero Eucalyptus deve ser conduzida de forma criteriosa, com a aplicação de brandos programas de secagem, caso contrário é inevitável a propensão a defeitos, como o desenvolvimento de colapso, rachaduras e empenamentos. Quanto à secagem da madeira de Hovenia dulcis Thunb (uva-do-Japão) são raras as informações sobre seu comportamento, independentemente do método utilizado. Susin et al. (2014) em estudos com a espécie, chama atenção para a aplicação da secagem em estufa solar, com intuito de reduzir o tempo e elevar a qualidade da madeira.

É justificável investigar à secagem em forno micro-ondas devido a carência de estudos deste cunho. Sua aplicação também se torna interessante contrastando madeiras distintas, porém altamente visados pelo setor madeireiro, caso do eucalipto, ou com potencial futuro, como a uva-do-japão. Nesse sentido, o objetivo deste estudo é avaliar a secagem da madeira abaixo do PSF em forno micro-ondas com diferentes potências de temperatura e comparar seu uso no processo de remoção de água em Hovenia dulcis e Eucalyptus tereticornis.

\section{Material e Métodos \\ Obtenção da madeira e confecção dos corpos de prova}

O material utilizado foi proveniente de um povoamento de Hovenia dulcis com 25 anos de idade oriundos da Fundação Estadual de Pesquisa Agropecuária - Centro de Pesquisa em Recursos Florestais (FEPAGRO FLORESTAS), localizado no Distrito da Boca do Monte em Santa Maria, RS. As árvores de Eucalyptus tereticornis, com 15 anos de idade, foram adquiridas no campus da Universidade Federal de Santa Maria, UFSM.

As toras foram cortadas na região do DAP e acondicionadas em laboratório por cerca de seis meses até a realização do desdobro. Então foram desdobradas em corpos de prova com dimensões de 40 × 50 × $60 \mathrm{~mm}$ (comprimento, largura e espessura), sem distinção entre cerne e alburno. Foram processadas 14 amostras de cada espécie sendo que destas, 7 foram submetidas a secagem no microondas a $50 \%$ da potência total e o restante à $100 \%$. No total utilizaram-se 28 amostras, divididas em 4 
tratamentos: T1 (50\% Hovenia dulcis), T2 (100\% Hovenia dulcis), T3 (50\% Eucalyptus tereticornis) e T4 (100\% Eucalyptus tereticornis).

\section{Tratamento com micro-ondas}

O equipamento utilizado para a elaboração dos tratamentos foi um forno micro-ondas convencional da marca LG com potência de $1450 \mathrm{~W}$. Os corpos de prova foram submetidos a diferentes potências $(50 \%$ e $100 \%$ do potencial total do aparelho) em intervalos de 2 minutos, até atingir o teor de umidade final (Tuf) de $12 \%$.

\section{Avaliações}

Antes da aplicação dos tratamentos de secagem da madeira em forno micro-ondas, o teor de umidade inicial de cada amostra foi calculado por meio da média aritmética. $\mathrm{O}$ teor de umidade foi determinado pela Equação (1), de acordo com o sugerido por Galvão e Jankowsky (1985).

$T U=\frac{P U-P S}{P S} \times 100$

Onde: TU: teor de umidade da amostra em porcentagem; $\mathrm{Pu}$ : peso úmido da amostra em gramas; PS: peso seco da amostra em gramas.

O teor de umidade das amostras foi monitorado por meio da Equação (2).

$T u a=\frac{P a(T u i+100)}{P i} \times 100$

Onde: Tua: teor de umidade atual (\%); Pa: peso atual da amostra (g); Pi: peso úmido da amostra (g); Tui: teor de umidade inicial $(\%)$.

Após a secagem por micro-ondas, as informações foram compiladas para elaboração da curva de secagem de acordo com a Equação (3) descrita por Santini (1980). A massa úmida inicial das amostras foi obtida em balança digital de precisão igual a $0,01 \mathrm{~g}$ enquanto as dimensões iniciais (largura, espessura e comprimento) foram obtidas com paquímetro digital com precisão de $0,01 \mathrm{~mm}$.

$T S=\frac{T u i-T u f}{t}$

Onde: $\mathrm{Ts}==$ taxa de secagem $(\% / \mathrm{min})$; Tui= teor de umidade inicial (\%); Tuf = teor de umidade final (\%); $\mathrm{t}=$ tempo de secagem decorrido (min.).

A avaliação da secagem foi feita por meio da análise do teor de umidade final e do gradiente de umidade, decorridas 24 horas do término da fase de resfriamento das cargas de secagem, conforme recomendado por Galvão e Jankowsky (1985).
Para determinação do gradiente de umidade após a secagem, cada amostra foi cortada em três seções A, B e C, com $2 \mathrm{~mm}$ de espessura cada (Figura 1).

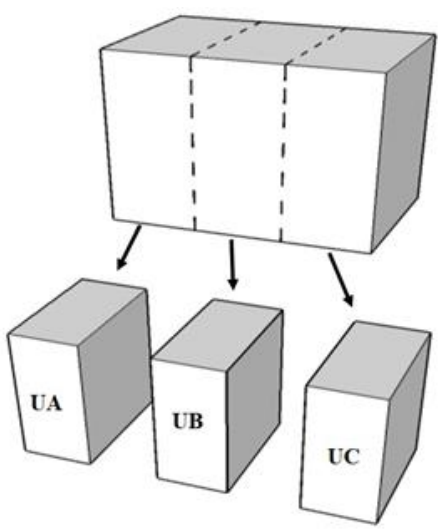

Figura 1. Esquema de obtenção das amostras para o cálculo do gradiente de umidade, onde UA umidade na superfície externa 1 da amostra; UC umidade na superfície externa 2 da amostra (\%) e UB umidade no interior da amostra.

Calculou-se o gradiente de umidade, entre B e A e entre B e C, de acordo com as Equações (4) e (5).

$$
\begin{aligned}
& \mathrm{G}_{1}=\mathrm{UB}-\mathrm{UA} \\
& \mathrm{G}_{2}=\mathrm{UB}-\mathrm{UC}
\end{aligned}
$$

Onde: $\mathrm{G}_{1}$ - gradiente de umidade $1(\%) ; \mathrm{G}_{2}-$ gradiente de umidade $2(\%)$; UB - umidade no interior da amostra (\%); UA - umidade na superfície 1 da amostra (\%); UC - umidade na superfície 2 da $\operatorname{amostra}(\%)$.

\section{Análise estatística}

$\mathrm{O}$ delineamento experimental utilizado foi o inteiramente casualizado (DIC). As análises estatísticas foram realizadas por meio do programa computacional Assistat 7.7. Para a análise dos diferentes parâmetros aferidos utilizou-se a análise de variância (teste $\mathrm{F}$ a $5 \%$ de significância), e nos casos em que a hipótese da nulidade foi rejeitada, aplicou-se o teste de Tukey (5\% de significância) para a diferenciação das médias.

\section{Resultados e discussão}

O maior teor de umidade inicial foi verificado para a madeira de Hovenia dulcis $(83,80 \%)$ quando comparado a Eucalyptus tereticornis $(33,16 \%)$. A discrepância nos resultados está atrelada há alguns fatores: à variação natural de umidade existente entre as madeiras recém cortadas ou "verdes" de distintas espécies, causada por características peculiares a cada uma delas, como a porcentagem de cerne e alburno (Engelund, 2012). Com a densidade básica 
da madeira de E. tereticornis (podendo chegar a 1 $\mathrm{g} / \mathrm{cm}^{3}$ segundo Nogueira et al. 2020) superior a $H$. dulcis (entre 0,55 e $0,61 \mathrm{~g} / \mathrm{cm}^{3}$, determinada por Motta et al. 2014; Eleotério et al. 2015; Candaten et al. 2017). Em geral, o teor de umidade inicial se apresenta inversamente correlacionado com a densidade (Silva, 2003). E ao tempo transcorrido de seis meses entre o corte das toras e a realização do experimento. Neste período, embora acondicionadas em laboratório, a água livre teve sua movimentação facilitada entre os espaços vazios na madeira de eucalipto, refletindo em rachaduras e colapsos (Jankowsky et al., 2003).

Para o teor de umidade final (Tuf), todos os tratamentos mostram-se estatisticamente semelhantes. Entretanto, pode-se observar valores absolutos de médias menores em Eucalyptus tereticornis (Tabela 1).

Tabela 1. Teor de umidade final (\%) na madeira de Hovenia dulcis e Eucalyptus tereticornis nas diferentes potências utilizadas para a condução da secagem.

\begin{tabular}{llll}
\hline \multicolumn{4}{c}{ Teor de umidade final (Tuf) } \\
\hline & $\mathbf{5 0 \%}$ & $\mathbf{1 0 0 \%}$ & Média \\
\hline Hovenia dulcis & $13,68(2,01)$ & $12,36(2,43)$ & $13,02 \mathrm{a}$ \\
\hline Eucalyptus tereticornis & $12,61(0,80)$ & $12,84(0,49)$ & $12,72 \mathrm{a}$ \\
\hline Média & $13,14 \mathrm{~A}$ & $12,60 \mathrm{~A}$ & \\
\hline
\end{tabular}

*Não significativo para interação entre fatores. Médias de cada fator na linha comparadas entre si pelo teste de Tukey à $5 \%$ de probabilidade, entre parênteses o desvio padrão médio.

A umidade final programada em $12 \%$ foi alcançada na maioria dos tratamentos, exceto em $\mathrm{T} 1$, $50 \%$ Hovenia dulcis, que mesmo após estabilização atingiu $13,68 \%$, indicando que a maior potência deve ser escolhida para secar peças desta espécie e assim utilizá-las com menores riscos de defeitos de secagem, decorrentes da falta de estabilização da umidade da madeira com o ambiente.

Na Tabela 2 é possível visualizar o tempo necessário para que os tratamentos alcançassem o teor de umidade final próximo a $12 \%$. Mesmo com o teor umidade inicial superior de $83,80 \%$, a madeira de Hovenia dulcis atingiu um teor de umidade médio final em menor tempo.

Tabela 2. Tempo de duração da secagem em forno micro-ondas das madeiras de Hovenia dulcis e Eucalyptus tereticornis nos diferentes tratamentos, até alcançar teor de umidade final de $12 \%$.

\begin{tabular}{ll}
\hline Tratamento & $\begin{array}{l}\text { Duração } \\
\text { (minutos) }\end{array}$ \\
\hline T1 - Hovenia dulcis 100\% & 27 \\
T2 - Hovenia dulcis 50\% & 48 \\
T3 - Eucalyptus tereticornis & 30 \\
100\% & \\
T4 - Eucalyptus tereticornis 50\% & 88 \\
\hline
\end{tabular}

Nos eucaliptos a rápida saída da umidade interna, principalmente da água de impregnação, é dificultada devido a sua constituição anatômica. As pontuações de pequeno diâmetro causam dificuldade ou impedimento do deslocamento de água, através da capilaridade ou na forma líquida no interior da madeira. A permeabilidade da madeira de eucalipto se torna ainda mais difícil devido à presença de tiloses que obstruem severamente o interior dos elementos vasculares (Silva, 2003).
Sobre o tempo de secagem em forno microondas, Oloyede e Groombridge (2000) reforçam que, se aplicado corretamente, o método é mais rápido e provavelmente mais barato a longo prazo após o desembolso inicial de custo de capital para equipamentos. A potencial economia de tempo de secagem por micro-ondas, comentam os autores, tende a ser o principal fator de motivação e interesse em sua aplicação à secagem de madeiras.

Outro fator importante a ser considerado refere-se ao acondicionamento das toras por seis meses, anterior a aplicação dos tratamentos. Caso esse período fosse aumentado e as condições de armazenamento melhoradas, principalmente a temperatura, a umidade relativa do ar e a velocidade do vento, a madeira poderia ter seu tempo de secagem em micro-ondas reduzido, ao passo que seu teor de umidade inicial, de 83,80\% em Hovenia dulcis e $33,16 \%$ em Eucalyptus tereticornis, seria reduzido o mais próximo possível do PSF.

A secagem ao ar livre, conforme colocação de Zen et al (2019), torna-se um método interessante que pode ser usado também de formas alternativas, como o método combinado (pré-secagem mais secagem artificial em estufa), onde a pré-secagem retira lentamente a água livre, diminuindo as tensões na madeira e posteriormente a remoção da água de impregnação é finalizada em estufa, evitando rachaduras e defeitos como o colapso.

$\mathrm{Na}$ aplicação do tratamento usando a potência em $50 \%$ para a madeira de Hovenia dulcis com umidade inicial de $83,80 \%$, houve o aumento do tempo transcorrido em duas vezes até o teor de umidade final. Essa relação é maior em Eucalyptus tereticornis, embora sua umidade inicial tenha sido menor $(33,16 \%)$, a potência de $50 \%$ acarretou aumento de mais que o dobro do tempo, assinalando a dificuldade de secagem das madeiras de eucalipto, também com essa técnica (Figura 2 e 3 ). 


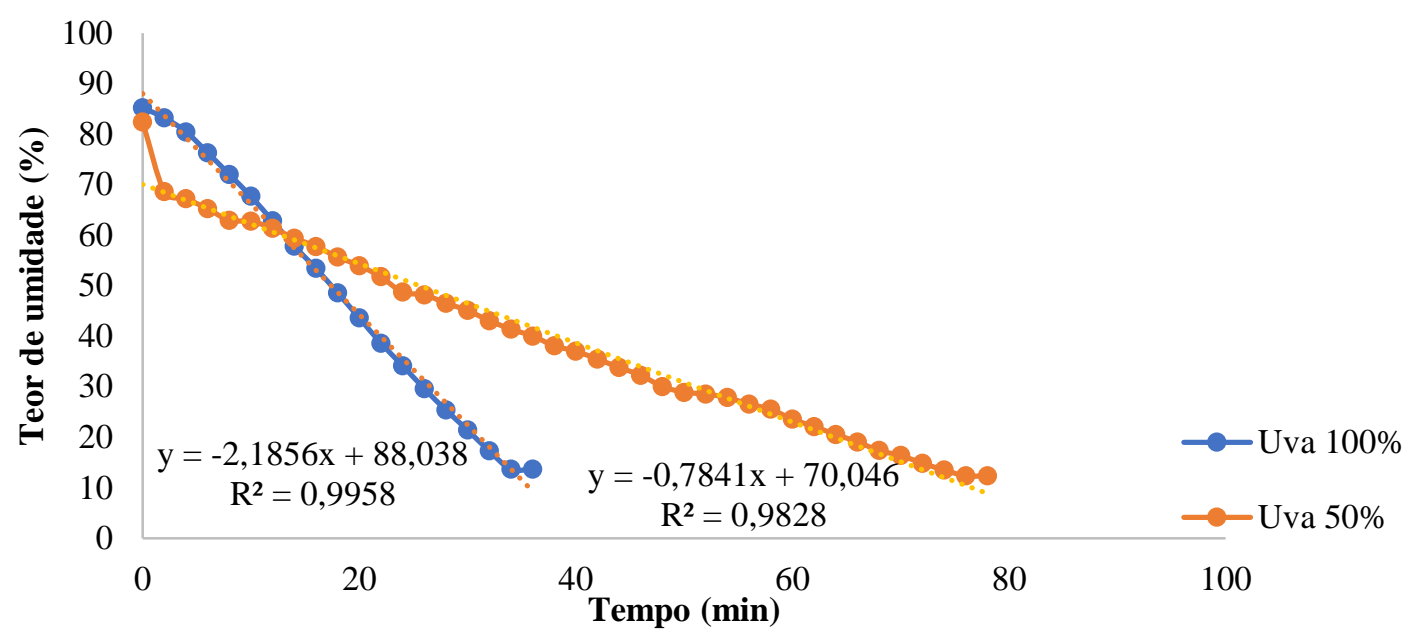

Figura 2. Curvas de secagem das amostras de madeira de Hovenia dulcis em função do teor de umidade inicial e tempo decorrido em horas nas diferentes potências do micro-ondas.

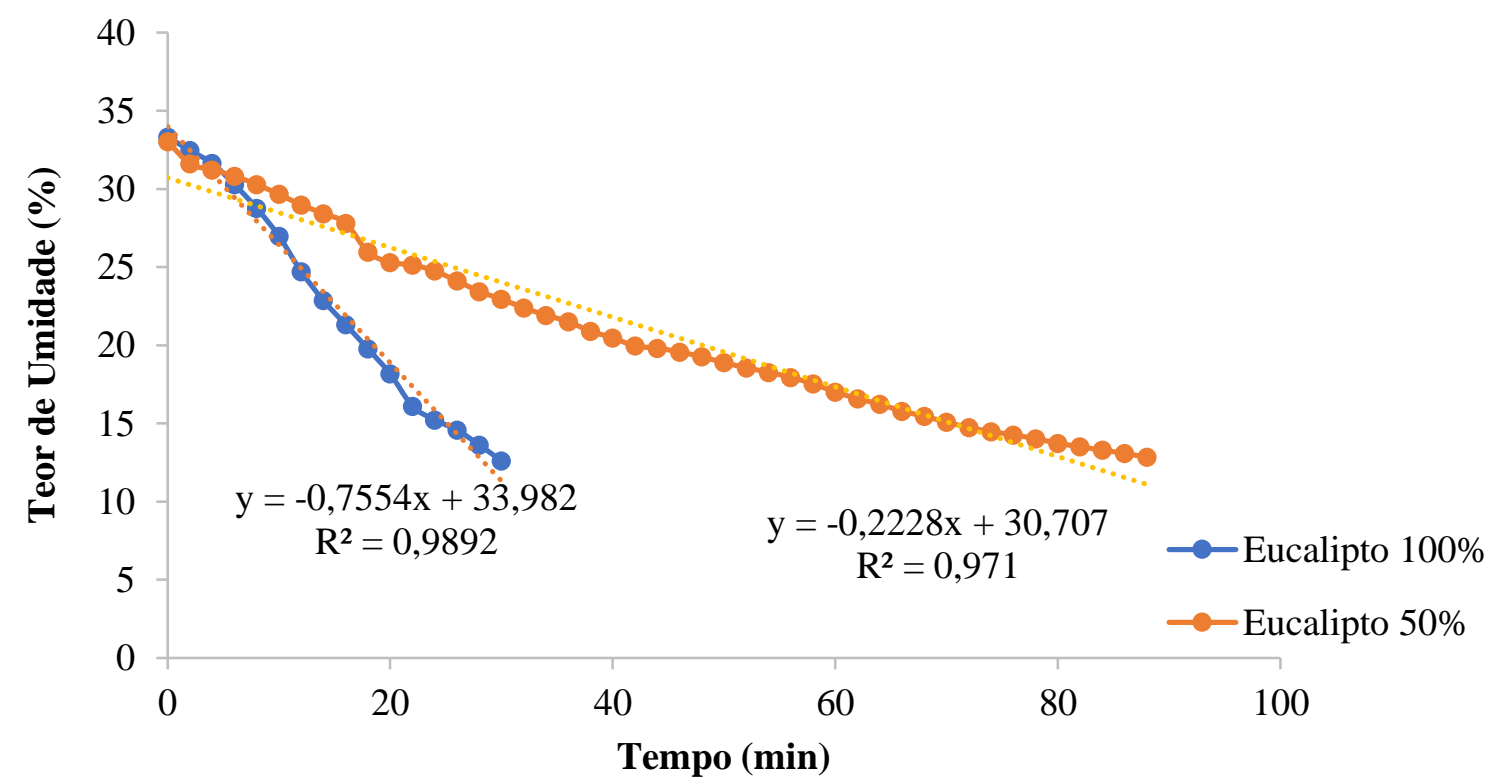

Figura 3. Curvas de secagem das amostras de madeira de Eucalyptus tereticornis em função do teor de umidade inicial e tempo decorrido em horas nas diferentes potências do micro-ondas.

As características da madeira exercem influência direta nos resultados obtidos e devem ser consideradas em todos os programas de secagem. Na anatomia de Hovenia dulcis, Motta et al. (2014) descrevem poros pouco abundantes $\left(6,8\right.$ poros $\left./ \mathrm{mm}^{2}\right)$, porém em grandes diâmetros $(114,7 \mu \mathrm{m})$. Juntamente as fibras curtas $(967,8 \mu \mathrm{m})$ e de paredes delgadas a espessas $(4,8 \mu \mathrm{m})$ tais caracteres seriam facilitadores do fluxo de água e aumento da permeabilidade durante a secagem. Já o Eucalyptus tereticornis é bem conhecido por apresentar, dentre tantas espécies do gênero, uma das madeiras mais resistentes, dura, com alta densidade básica (Nogueira et al. 2020). Assim, há de se esperar que a madeira com maior densidade básica tenha células anatômicas com paredes celulares mais espessas e menos espaços internos para circulação da água, refletindo na demora e dificuldade de secagem (Zanuncio et al. 2016).

Como reportado por Tsoumis (1991) o comportamento dos tempos de secagem durante os tratamentos também pode ter sido influenciado por variações encontradas na amostra, como por exemplo, quantidade de cerne da madeira, composição química, entre outros. Para maior confiabilidade nesta correlação, nos próximos experimentos é indicado a separação das peças em cerne e alburno anteriormente a secagem em forno micro-ondas.

Ainda, nas Figuras 2 e 3, é possível visualizar que o processo de secagem em forno micro-ondas proporciona a perda de água de maneira constante, independente se considerada a água livre, ou de impregnação. Tal comportamento é diferente 
das secagens tradicionais, em estufa ou ar livre, que geralmente apresentam curvas exponenciais ou em "J invertido". Na secagem da madeira de Tectona grandis e Acacia mangium ao ar livre, BRAZ, et al., (2015) inferem que acima do PSF, a velocidade de secagem permanece constante, desde que a temperatura e umidade relativa do ar permaneçam estáveis. Já abaixo do PSF, a água retida na madeira se move mais lentamente. Sendo assim, a perda de umidade no início do processo é bem mais rápida, como também pode ser visto na curva de secagem típica da madeira ao ar livre, do trabalho realizado por Eleotério et al., (2015).
Os resultados assinalam que o processo de secagem da madeira em forno micro-ondas é capaz de remover a água de maneira homogênea e constante, possibilitando a criação de programas de secagem específicos, que culminem em menor tempo de processo e defeitos nas peças secas.

A menor taxa de secagem de $0,22 \%$ de umidade por minuto foi observada para a madeira de Eucalyptus tereticornis utilizando $100 \%$ da potência do forno micro-ondas (Tabela 3). Nas maiores potências também, para as duas espécies, a água flui mais rapidamente para o exterior da madeira.

Tabela 3. Taxas de secagem (\%/min) das madeiras de Hovenia dulcis e Eucalyptus tereticornis nas diferentes potências utilizadas para a condução da secagem.

\begin{tabular}{lcccc}
\hline & \multicolumn{4}{c}{ Tratamentos } \\
\hline Hovenia dulcis & $\mathbf{5 0 \%}$ & Tempo (min) & $\mathbf{1 0 0 \%}$ & Tempo (min) \\
\hline Eucalyptus tereticornis & $1,98 \mathrm{aA}(2,05)$ & 48 & $0,89 \mathrm{aB}(2,87)$ & 27 \\
\hline
\end{tabular}

* Médias seguidas pela mesma letra maiúscula na linha e minúscula na coluna não diferem entre si pelo teste $\mathrm{t}$ à $5 \%$ de probabilidade, entre parênteses o desvio padrão das amostras.

O melhor desempenho na taxa de secagem foi verificado para Hovenia dulcis, mesmo com a sua madeira apresentando mais que o dobro de umidade em relação ao Eucalyptus tereticornis. Os resultados afirmam a dificuldade prática na secagem de eucaliptos. A impermeabilidade destas espécies, principalmente em teores de umidade abaixo do PSF, causa os temíveis defeitos de secagem, como colapsos e fendas (Jankowsky et al., 2003).

$\mathrm{Na}$ Tabela 4 estão dispostos os valores médios dos gradientes de umidade de cada espécie em

Tabela 4. Médias dos gradientes de umidade para as madeiras de Hovenia dulcis e Eucalyptus tereticornis nas diferentes potências utilizadas para a condução da secagem

\begin{tabular}{ccc}
\hline Tratamentos & Gradiente de Umidade (\%) \\
\cline { 2 - 3 } & 1 & 2 \\
\hline Hovenia dulcis 100\% & $0,63 \mathrm{a}$ & $0,00 \mathrm{ab}$ \\
Hovenia dulcis 50\% & $0,49 \mathrm{a}$ & $-0,03 \mathrm{ab}$ \\
Eucalyptus tereticornis 100\% & $-1,36 \mathrm{ab}$ & $-1,82 \mathrm{~b}$ \\
Eucalyptus tereticornis 50\% & $-1,63 \mathrm{ab}$ & $-1,88 \mathrm{~b}$ \\
\hline
\end{tabular}

* Letras iguais indicam que, ao nível de 5\% de significância, não há diferença entre as médias nas colunas.

Normalmente em um material com alta quantidade de água livre, como nas madeiras estudadas, muitas moléculas são orientadas aleatoriamente (Gao 2010), porém o campo elétrico tende a alinhar com a rotação campo - dipolo. Com as flutuações do campo, estes processos de alinhamento e relaxamento ocorrem milhões de vezes por segundo, convertendo a energia elétrica em energia potencial e em seguida em calor (Cavalcante et al. 2016). Desta maneira, a secagem com o emprego de micro-ondas ocorre de maneira distinta em relação a secagem convencional.

Uma das principais dúvidas da indústria de secagem convencional da madeira é como retirar a função do tratamento utilizado. No caso da madeira de Eucalyptus tereticornis os gradientes apresentamse negativos, indicando que a parte interna das amostras estava com umidade inferior em relação às partes externas. Esse processo pode ser explicado porque uma das principais características da secagem por micro-ondas é o aquecimento de dentro para fora, gerando calor diretamente no centro da madeira. 
do micro-ondas; como reduzir os custos com energia até que se torne economicamente viável; como migrar de um projeto em escala piloto e extrapolar para unidades capazes de secar grandes quantidades de madeiras.

\section{Conclusões}

A madeira de Eucalyptus tereticornis apresentou maior dificuldade de secagem em comparação com a madeira de Hovenia dulcis em ambas as potências de temperaturas. Os gradientes de umidade foram negativos para a madeira de Eucalyptus tereticornis, evidenciando que o uso do forno micro-ondas, por secar do exterior para o interior da peça, pode ser recomendado em madeiras de difícil secagem e com alta incidência de defeitos.

\section{Referencias}

Duarte TEPN, Angeoletto F, Santos JWMC, Silva Braz, RL, Duarte, APC, Oliveira, JTS, Motta, JP, Rosado, AM (2015). Curva Característica de Secagem da Madeira de Tectona grandis e Acacia mangium ao Ar Livre. Floresta e Ambiente, 22(1):117-123. doi10.1590/2179-8087.037913

Candaten L, Zanatta T, Rosa M, Trevisan R, Costa HWD (2017) Variação axial da massa específica básica de Hovenia dulcis. Enciclopédia biosfera, 14(26): 729-736. doi: 10.18677/EnciBio_2017B67

Cavalcante AA, Naveiro RM, Costa SS (2016) Secagem da Madeira de Louro Preto (Nectandra cuspidata) em Estufa de Micro-ondas. Floresta $e$ Ambiente, 23(3): 427-434. doi: 10.1590/21798087.012412

Eleotério JR, Pelles GC, Commanduli MJ (2015) Variação longitudinal da massa específica e da casca da madeira de Hovenia dulcis Thunberg. Ciência da Madeira: 6(2): 122-128. doi: 10.12953/21776830/rcm.v6n2p122-128.

Eleoterio, J R, Bagattoli, TR, Hornburg, KF, Kirchheim da Silva, CM (2015) Secagem drástica de madeiras de Eucalyptus e Corymbia fornece informações para a elaboração de programas de secagem. Pesquisa Florestal Brasileira, 35(84): 451-547. Disponível em: $<$ https://pfb.cnpf.embrapa.br/pfb/index.php/pfb/arti cle/view/696>.

Engelund ET, Thygesen LG, Svensson S, Hill CAS (2013) A critical discussion of the physics of woodwater interactions. Wood Sci Technol 47:141161.doi: 10.1007/s00226-012-0514-7

Galvão AP, Jankowsky IP (1985) Secagem racional da madeira. São Paulo: Nobel. 111p.
Guella AF (2016) Madeira: Desafio e tendências. Revista da Madeira, Curitiba, 2016.

Harris GA, Torgovnikov G, Vinden P, Brodie GI, Shaginov A (2008) Microwave Pretreatment of Backsawn Messmate Boards to Improve Drying Quality: Part 1, Drying Technology: An International Journal, 26(5): 579-584. doi: $10.1080 / 07373930801944770$

Jankowsky IP, Santos GRV, Andrade A (2003) Secagem da madeira serrada de eucalipto. Piracicaba, n. 199, (IPEF. Circular técnica, n. 199).

Motta JP, Oliveira JTS, Braz RL, Duarte ACP, Alves RC (2014) Caracterização da madeira de quatro espécies florestais. Ciência Rural, 44(12): 21862192. doi: $10.1590 / 01038478 \mathrm{cr} 20130479$

Mogueira MCJA, Araujo VA, Vasconcelos JS, Christoforo AL, Lahr FAR (2020) Sixteen properties of Eucalyptus tereticornis wood for structural uses. Biosci. J., 36(2): 449-457. doi: 10.14393/BJv36n2a2020-45169

Oloyede A, Groombridge, P (2000) The influence of microwave heating on the mechanical properties of wood. J. Mater. Process. Technol. 100: 67-73.

Santini EJ (1980) Influência de temperatura na taxa de secagem e contração da madeira de Pinus elliottii proveniente de plantios de rápido crescimento. FUPEF. Série Técnica, 5. 15p.

Santini EJ, Talgatti M, Silveira AG, Haselein CR, Azambuja, RR (2016) Secagem e qualidade da madeira serrada de Hovenia dulcis. Sci. Agrar. Parana., 15(4): 460-463. doi:10.18188/19831471/sap.v15n4p460-463

Silva JC (2003) Secagem da Madeira de Eucalipto. Revista da madeira, ed 75.

Susin F, Santini EJ, Stangerlin DM, Moaris WWC, Melo RR (2014) Taxa de secagem e qualidade da madeira serrada de Hovenia dulcis submetida a dois métodos de secagem. Floresta e Ambiente, 21(2): 243-250. doi: 10.4322/floram.2014.016

Torgovnikov G, Vinden P (2010) Microwave wood modification technology and its applications. Forest Products Journal, 60(2): 84-92. doi: 10.13073/00157473-60.2.173.

Tsoumis G (1991) Science and technology of wood. New York: Chapman \& Hall; 494p.

Zanuncio, AJV, Carvalho, AG, Damásio, RAP, Oliveira BS, Carneiro ACO, Colodette JL (2016). Relationship between the anatomy and drying in 
Talgatti et al.

Eucalyptus grandis x Eucalyptus urophylla wood. Revista Árvore, 40(4): 723-729. doi: 10.1590/010067622016000400016

Zen, LR, Monteiro, TC, Schaeffera, W, Kaminskia, JM, Klitzkea, RJ (2019). Secagem ao ar livre da madeira serrada de eucalipto. Journal of Biotechnology and Biodiversity, 7(2): 291-298. doi: 10.20873/jbb.uft.cemaf.v7n2.zen 